Editor's Note: These short reviews of a recent paper in the Journal, written exclusively by graduate students or postdoctoral fellows, are intended to mimic the journal clubs that exist in your own departments or institutions. For more information on the format and purpose of the Journal Club, please see http://www.jneurosci.org/misc/ifa_features.shtml.

\title{
Policing the Police: Astrocytes Modulate Microglial Activation
}

\author{
Andy Y. Shih, ${ }^{1,2,3}$ Herman B. Fernandes, ${ }^{1,2,3}$ Fiona Y. Choi, ${ }^{1,2,3}$ Michael G. Kozoriz, ${ }^{4}$ Yingru Liu, ${ }^{1,3}$ Ping Li, ${ }^{1,2}$ \\ Catherine M. Cowan, ${ }^{2,3}$ and Andis Klegeris ${ }^{2}$ \\ ${ }^{1}$ Graduate Program in Neuroscience, ${ }^{2}$ Kinsmen Laboratory of Neurological Research, ${ }^{3}$ Brain Research Centre, and ${ }^{4}$ Department of Cellular and \\ Physiological Sciences, University of British Columbia, Vancouver, British Columbia, Canada V6T 1Z3 \\ Review of Min et al. (http://www.jneurosci.org/cgi/content/full/26/6/1880)
}

Within hours after an acute brain injury such as a stroke, the brain mounts an inflammatory response involving activation of local microglia/astrocytes and infiltration of macrophages from circulation. The inflammatory process is a doubleedged sword, because increased production of reactive oxygen and nitrogen species (ROS/RNS) by immune cells can cause secondary damage up to weeks after injury, yet the subsequent remodeling of brain tissue requires the efficient containment and removal of dead tissue. The recent paper by Min et al. (2006) in The Journal of Neuroscience furthers our understanding of how the brain intrinsically controls inflammation during injury, providing important clues on how damage caused by inflammation can be minimized while beneficial effects are maximized.

Microglia are resident immune cells of the brain and an important component of the brain inflammatory response. In previous studies, the authors demonstrated that microglial activation could be regulated by the oxidative state of the cell. Exposure to inflammatory

\footnotetext{
Received March 2, 2006; accepted March 4, 2006.

We thank the Brain Research Centre, in particular Dr. Max Cynader, Swarni Sunner, Melissa Waddell, and Chris Crossfield, for generous support of our Stroke and Neurodegenerative Disease Journal Club. We also thank Dr. Timothy H. Murphy for helpful comments.

Correspondence should be addressed to Andy Y. Shih, 4835-2255 Wesbrook Mall, University of British Columbia, Vancouver, BC, Canada V6T $1 Z 3$. E-mail: ashih@interchange.ubc.ca.

DOI:10.1523/JNEUROSCI.0936-06.2006

Copyright $\odot$ 2006 Society for Neuroscience $\quad$ 0270-6474/06/263887-02\$15.00/0
}

cytokines, for example, increased intracellular ROS/RNS production and shifted microglia to an activated state. Conversely, application of antioxidants suppressed this effect. In the current study, they show that soluble astrocytederived factor(s) can induce antioxidant gene expression in microglia in vitro and thus modulate microglial redox status and activation state. The authors hypothesize that this astrocyte-microglial interaction could contribute to regulation of brain inflammation in vivo.

To examine whether a soluble factor(s) released from astrocytes modulates microglia ROS/RNS production, the authors harvested culture media previously incubated with confluent astrocyte cultures for $3 \mathrm{~d}$ [astrocyteconditioned media (ACM)]. Separate microglial cultures were then pretreated with ACM, before exposure to $\mathrm{H}_{2} \mathrm{O}_{2}$, a reactive molecule capable of generating ROS. Microglial ROS production was significantly reduced with ACM pretreatment (6-12 h) [Min et al. (2006), their Fig. $1 D$ (http://www.jneurosci. org/cgi/content/full/26/6/1880/F1)]. It is important to note that the authors used serum-free culture media to collect ACM for $3 \mathrm{~d}$. We suspect that astrocytes cultured for this period may be in an activated state, perhaps simulating activated astrocytes within an in vivo injury site.

ACM might exert its effect by increasing the expression of microglial antioxidant enzymes. Indeed, microglia treated with ACM show increased mRNA and protein expression of the heme-catabolizing enzyme, heme oxygenase-1 (HO-1) [Min et al. (2006), their Fig. 2A,B (http://www. jneurosci.org/cgi/content/full/26/6/1880/ F2) ]. HO-1 activity may reduce microglial ROS production by generating biliverdin, that can be further reduced by biliverdin reductase to bilirubin, a potent antioxidant (Fig. 1A) [Min et al. (2006), their Fig. 2C (http://www.jneurosci.org/ cgi/content/full/26/6/1880/F2)]. Additionally, HO-1-dependent release of iron ions stimulates expression of ferritin, an iron-sequestering protein that can prevent conversion of $\mathrm{H}_{2} \mathrm{O}_{2}$ to hydroxyl radical through the Fenton reaction. Importantly, HO-1 and ferritin are coregulated by the basic leucine zipper transcription factor Nrf2 (Alam et al., 1999; Kwak et al., 2001).

Nrf2 reduces cellular oxidative load by coordinating global expression of genes involved in ROS scavenging, glutathione biosynthesis, detoxification of xenobiotics, and NADPH generation. Normally bound to Keap1 in the cytoplasm, Nrf2 translocates to the nucleus to initiate gene expression in response to oxidative stress or similar signals (Fig. 1A) (Motohashi and Yamamoto, 2004). Accordingly, the authors found that ACM stimulated nuclear translocation of Nrf2 in microglia [Min et al. (2006), their Fig. 3A (http:// www.jneurosci.org/cgi/content/full/26/6/ 1880/F3)]. The Nrf2 transcription complex specifically recognizes a 51 bp DNA element, the antioxidant response ele- 
ment (ARE), located in the promotor of all Nrf2 gene targets. The mouse HO-1 gene promotor carries three such AREs (Alam et al., 1999). The authors show that ACM augmented ARE-dependent gene expression in microglia using mobility shift assays and luciferase expression reporter assays driven by the mouse HO-1 promotor [Min et al. (2006), their Fig. 4 (http://www.jneurosci.org/cgi/content/full/ 26/6/1880/F4)]. Although the authors have convincingly demonstrated the upregulation of ARE-dependent gene expression, as mentioned in their Discussion, they have not ruled out the involvement of other ARE-binding transcription factors [i.e., C/EBP (CCAAT/ enhancer-binding protein), SP1 (specificity protein 1), and Nrf1]. This possibility could be examined using Nrf2 knock-out cultures or with acute small interfering RNA (siRNA) knock-down of Nrf2.

The authors hypothesize that ACMmediated Nrf2 activation suppresses the microglial inflammatory response. To test this idea, they used a more physiologically relevant stimulus, the proinflammatory cytokine, interferon- $\gamma$ (IFN- $\gamma)$. Consistent with their hypothesis, ACM pretreatment attenuated IFN- $\gamma$-induced ROS/ RNS production and inducible nitric oxide synthase expression in microglia [Min et al. (2006), their Fig. 5 (http://www. jneurosci.org/cgi/content/full/26/6/1880/ F5)]. Exogenous bilirubin and $\mathrm{FeSO}_{4}$ (an iron donor) mimicked the effect of ACM, and CORM (CO-releasing molecule) also partially mimicked ACM, suggesting that $\mathrm{HO}-1$ activity may be sufficient to explain their findings [Min et al. (2006), their Fig. 6 (http://www.jneurosci.org/cgi/content/ full/26/6/1880/F6)]. However, other Nrf2regulated pathways have not been ruled out (Fig. 1A). In fact, it is conceivable that microglial redox state is effectively controlled by the coordinated action of multiple Nrf2regulated antioxidant enzymes (i.e., superoxide dismutase and glutathione production enzymes) rather than $\mathrm{HO}-1$ alone (Shih et al., 2003). Chemical inhibition or siRNA knock-down of HO-1 would help solve this question.

To identify the Nrf2-inducing factor found in ACM, the authors narrowed down the possibilities by showing that the molecule is heat labile and smaller than 3 kDa [Min et al. (2006), their Fig. 7 (http:// www.jneurosci.org/cgi/content/full/26/6/ 1880/F7)]. TGF- $\beta$, a molecule known to induce HO-1 gene expression, was tested as a candidate. However, exogenous TGF- $\beta$ at the concentrations in ACM

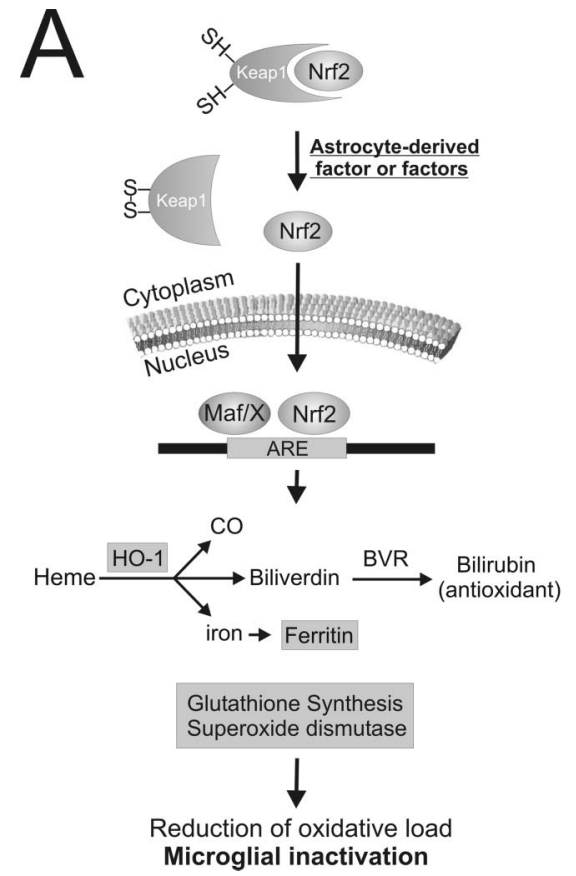

B

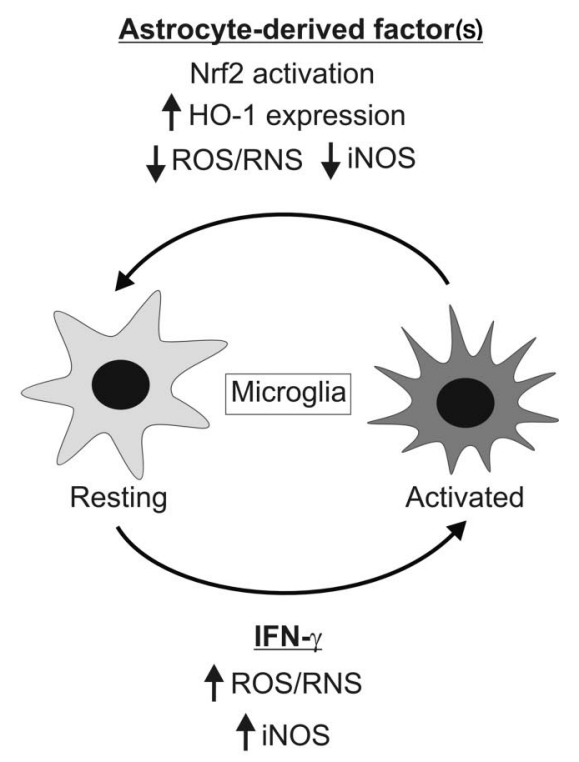

Figure 1. A, Schematic diagram showing Nrf2 dissociation from Keap1 and induction of ARE-mediated gene expression. Nrf2 is normally localized to the cytoplasm, tethered to an actin-bound regulatory protein, Keap1. Increased oxidative stress directly modifies key sulfhydryl group interactions in the Nrf2-Keap1 complex allowing Nrf2 dissociation, nuclear translocation, and participation in a transcription complex with other small Maf proteins (Motohashi and Yamamoto, 2004). Min et al. (2006) describe a soluble astrocyte-derived factor that promotes Nrf2 nuclear translocation. In addition to H0-1, Nrf2 regulates many other antioxidant enzymes including ferritin, glutathione synthesis enzymes, and the free radical scavenger superoxide dismutase (shown in shaded boxes). BVR, Biliverdin reductase. $\boldsymbol{B}$, Resting microglia can be activated by exposure to the proinflammatory cytokine IFN- $\gamma$. Activation leads to an increased production of ROS/RNS and expression of iNOS. Astrocytes provide negative regulation by releasing an unidentified factor(s) that induces microglial Nrf2 activity and increases expression of antioxidant enzyme H0-1. The antioxidant products of H0-1 suppress ROS/RNS production and return microglia to the resting state.

failed to induce HO-1. Nevertheless, TGF- $\beta$ may still prove to be critical in vivo particularly if parallel signals are absent in the cultures.

Microglial inflammatory responses are essential to the wound repair process but also mediate collateral damage of neighboring cells by releasing ROS/RNS. The conclusions of Min et al. (2006) are an important step toward understanding how this delicate process is controlled in the brain. Their results indicate that astrocytes can regulate microglial activity by stimulating their Nrf2-dependent antioxidant gene expression, perhaps providing a negative feedback response to titrate the inflammatory reaction induced by proinflammatory mediators (Fig. $1 B$ ). Min et al. (2006) are also among the first to demonstrate a role for Nrf2 in controlling inflammation in brain using in vitro preparations. However, Nrf2 activity may be an important pathway for regulation of inflammatory responses throughout the entire body. Notably, loss of Nrf2 function in vivo exacerbates inflammation in skin and lungs (Lee et al., 2005).

\section{References}

Alam J, Stewart D, Touchard C, Boinapally S, Choi AM, Cook JL (1999) Nrf2, a Cap'n'Collar transcription factor, regulates induction of the heme oxygenase-1 gene. J Biol Chem 274:26071-26078.

Kwak MK, Itoh K, Yamamoto M, Sutter TR, Kensler TW (2001) Role of transcription factor $\mathrm{Nrf} 2$ in the induction of hepatic phase 2 and antioxidative enzymes in vivo by the cancer chemoprotective agent, $3 \mathrm{H}$-1,2-dimethiole3-thione. Mol Med 7:135-145.

Lee JM, Li J, Johnson DA, Stein TD, Kraft AD, Calkins MJ, Jakel RJ, Johnson JA (2005) Nrf2, a multi-organ protector? FASEB J 19:1061-1066.

Min K-J, Yang M-s, Kim S-U, Jou I, Joe E-h (2006) Astrocytes induce hemeoxygenase-1 expression in microglia: a feasible mechanism for preventing excessive brain inflammation. J Neurosci 26:1880-1887.

Motohashi H, Yamamoto M (2004) Nrf2-Keap1 defines a physiologically important stress response mechanism. Trends Mol Med 10:549-557.

Shih AY, Johnson DA, Wong G, Kraft AD, Jiang L, Erb H, Johnson JA, Murphy TH (2003) Coordinate regulation of glutathione biosynthesis and release by Nrf2-expressing glia potently protects neurons from oxidative stress. J Neurosci 23:3394-3406. 